\title{
Abstract zu: Der Neue Midcult Vom Wandel populärer Leseschaften als Herausforderung der Kritik
}

\author{
Erschienen in: Unterstellte Leseschaften \\ Von: Moritz Baßler
}

Der ausdifferenzierte Buchmarkt und die Möglichkeiten der sozialen Medien verändern nicht nur den Diskurs über Gegenwartsliteratur, sondern auch diese Literatur selbst. In dem Maße, wie ,Stilgemeinschaften normalisierten Spektakels' (Jochen Venus) zu den zentralen Rezeptions- und Wertungsinstanzen werden, verlieren die alten Gatekeeper in Kritik, Feuilleton und Universität ihre Funktion und müssen sich neu orientieren. Waren ihre professionellen Analysen und Wertungen immer noch heuristisch auf die eine Literatur bezogen, scheint unter den beschriebenen Bedingungen ein Zerfall in kaum noch miteinander vermittelte Leseschaften stattzufinden, wie er ähnlich im Bereich der Pop-Musik schon lange zu beobachten ist.

Unter dem ökonomischen Begriff der ,ästhetischen Genuinität‘ lassen sich diese Prozesse neu fassen: Nach dem Auswahlkriterium ,entspricht mir/entspricht mir nicht‘ entstehen tendenziell geschlossene Gemeinschaften mit spezifischen ästhetischen Erwartungen. Man kennt das aus den nerdigen Gefilden z. B. von Fantasy oder Science Fiction. Der Markt und die neuen Medien sorgen nun für erfolgreiche Rückkopplung von kultureller Produktion und Rezeption. So verhält es sich bereits im Midcult (Umberto Eco) eines Populären Realismus, der leicht goutierbare Romane mit schweren Kunstund Bedeutungszeichen anreichert und so ein Lesepublikum befriedigt, das unterhalten werden, aber auch an Hochkultur partizipieren möchte. Eine professionelle Kritik daran (Eco spricht von ,struktureller Lüge') läuft tendenziell ins Leere, weil Angebot und Nachfrage perfekt aufeinander abgestimmt sind und die Leseschaft die Normen jeder Kritik von außen als inadäquat zurückweist. Vorwürfe etwa des Elitismus und der Unzuständigkeit werden laut (z. B. in der Debatte um Takis Würgers Stella).

Zunehmend ist zu beobachten, dass die entsprechenden Stilgemeinschaften, von nerdigen Fans über emanzipatorische Minderheiten bis hin zum Mainstream, in diese ästhetische Genuinität nicht nur ästhetische, sondern auch ethische oder weltanschauliche Positionen und Normen einbeziehen. Damit bleibt die Abgeschlossenheit der Gemeinschaften und das Fehlen von Instanzen, die zwischen innen vermitteln, nicht länger ein bloß ästhetisches Problem. Interessant ist auch, was für literarische Qualitäten solche Stilgemeinschaften erwarten und damit hervorbringen: Positiv validiert werden vor allem Bücher, die die ,richtigen‘ Themen in einer 
identifikationsfähigen Form präsentieren. Dazu gehört insbesondere auch der ,richtige‘ Autor/die ,richtige‘ Autorin, die die entsprechende Position beglaubigen kann. Hier entsteht, so die These, ein Neuer Midcult, der inzwischen auch schon auf die Universität übergreift (diskutiert wird der Fall der Literaturwissenschaftlerin Amy Hungerford). Wie soll man sich als Literaturprofi dazu verhalten?

Auf dem Workshop ,Unterstellte Leseschaften‘ hat Moritz Baßler gemeinsam mit Julika Griem einen Vortrag gehalten. Er hat seinen Part zu einem Essay ausgebaut, der in POP - Kultur und Kritik (18/Frühling 2021) publiziert wurde.

Der Link verweist auf die Homepage der Zeitschrift: https://poppzeitschrift.de/2021/04/21/pop-kultur-und-kritik-heft-18-fruehling-2021inhaltsuebersicht-autordatum21-4-2020-datum/

SUGGESTED CITATION: Baßler, Moritz: Abstract zu: Der neue Midcult. Vom Wandel populärer Leseschaften als Herausforderung der Kritik, in: KWI-BLOG, [https://blog.kulturwissenschaften.de/abstract-zu-der-neue-midcult/], 21.07.2021

\section{DOI: https://doi.org/10.37189/kwi-blog/20210721-0830}

DuEPublico

Duisburg-Essen Publications online

\section{DEUSISB BU R G}

offen im Denken

Ub $\mid \begin{aligned} & \text { universitäts } \\ & \text { bibliothek }\end{aligned}$

Dieser Text wird via DuEPublico, dem Dokumenten- und Publikationsserver der Universität Duisburg-Essen, zur Verfügung gestellt. Die hier veröffentlichte Version der E-Publikation kann von einer eventuell ebenfalls veröffentlichten Verlagsversion abweichen.

DOI: $\quad$ 10.37189/kwi-blog/20210721-0830

URN: urn:nbn:de:hbz:464-20210721-183325-6 